\title{
Ethnomedicinal study of plants used in villages around Kimboza forest reserve in Morogoro, Tanzania
}

\author{
Ezekiel $\mathrm{Amri}^{{ }^{*}}$ and Daniel P Kisangau ${ }^{2}$
}

\begin{abstract}
Background: An ethnomedicinal study was conducted to document medicinal plants used in the treatment of ailments in villages surrounding Kimboza forest reserve, a low land catchment forest with high number of endemic plant species.

Methods: Ethnobotanical interviews on medicinal plants used to treat common illnesses were conducted with the traditional medical practitioners using open-ended semi -structured questionnaires. Diseases treated, methods of preparation, use and habitat of medicinal plants were recorded.

Results: A total of 82 medicinal plant species belonging to 29 families were recorded during the study. The most commonly used plant families recorded were Fabaceae (29\%), Euphorbiaceae (20\%), Asteraceae and Moraceae (17\% each) and Rubiaceae (15\%) in that order. The most frequently utilized medicinal plant parts were leaves (41.3\%), followed by roots (29.0\%), bark (21.7\%), seeds (5.31\%), and fruits (2.6\%). The study revealed that stomach ache was the condition treated with the highest percentage of medicinal plant species (15\%), followed by hernia (13\%), diarrhea (12), fever and wound (11\% each), and coughs (10\%). Majority of medicinal plant species (65.9\%) were collected from the wild compared to only $26.7 \%$ from cultivated land.

Conclusions: A rich diversity of medicinal plant species are used for treating different diseases in villages around Kimboza forest reserve, with the wild habitat being the most important reservoir for the majority of the plants. Awareness programmes on sustainable utilization and active involvement of community in conservation programmes are needed.
\end{abstract}

Keywords: Ethnobotany, Medicinal plants, Kimboza forest, Conservation

\section{Background}

Kimboza forest reserve has 13 recorded endemic plant species making it the richest lowland forest in East Africa. The forest reserve has valuable contribution to biological and gene pool conservation, and together with other mountain ranges of Morogoro region form part of the Eastern Highlands of Tanzania with about 200 endemic plant species $[1,2]$. The uses of plants in the indigenous cultures particularly of developing countries, are numerous and diverse, forming an important socio-economic base including their use as medicine [3]. People

\footnotetext{
* Correspondence: ezekielamri@yahoo.com

'Department of Science and Laboratory Technology, Dar es Salaam Institute of Technology, P. O. Box 2958, Dar es Salaam, Tanzania

Full list of author information is available at the end of the article
}

generally depend on nearby forests for fuel wood, timber and medicine. Medicinal plants therefore have important contribution in the primary healthcare systems of local communities as the main source of medicines for the majority of the rural population $[4,5]$.

The World Health Organisation (WHO) estimates that up to $80 \%$ of the world's population in developing countries depend on locally available plant resources for their primary healthcare, since western pharmaceuticals are often expensive, inaccessible or unsuitable [6]. Further, in this decade, the world is experiencing an increasing rate of resistance by pathogens to some of the synthetic drugs, as well as the struggle against some chronically complex and uncontrolled infections such as Cancer and HIV/AIDS. There is therefore need to study

\section{Biomed Central}


and validate ethnomedicines for wider acceptance, recognition and utilization by all stakeholders in the society. However, overtime, ethnomedicinal knowledge has been undermined by mortality of resource persons, extinction of plant resources, inadequate scientific research and poor documentation [7]. The aim of the present study was therefore to document ethnomedicinal information of plants used by indigenous people in villages surrounding Kimboza forest reserve. The generated information will be used in future to explore ways of sensitizing the community on the sustainable utilization of the forest resources so as to minimize their genetic loss.

\section{Methods}

\section{Study area}

The study area is about $60 \mathrm{~km}$ from Morogoro Municipal located between $06^{\circ} 59^{\prime}-7^{\circ} 02^{\prime} \mathrm{S}$ and $37^{\circ} 47^{\prime}-37^{\circ}$ 49'E. An ethnobotanical survey for medicinal plants was conducted in the following villages: Changa, Kibangile, Mwarazi and Uponda which surround Kimboza forest reserve (Figure 1). The communities adjacent to the forest have access right over the forest as stipulated in the village forest management plan by-laws.

\section{Data collection}

Ethnobotanical surveys were carried out to obtain information on medicinal plants traditionally used to treat various ailments in the study area. With the help of local administrative officers, Participatory Rural Appraisal (PRA) was employed to identify key respondents [8]. Ethnobotanical data was collected through individual and Focus Group Discussion (FGD) interviews using semi-structured open-ended questionnaires. Interviews were conducted in Swahili or the local Kiluguru language. Field excursions were also conducted with the

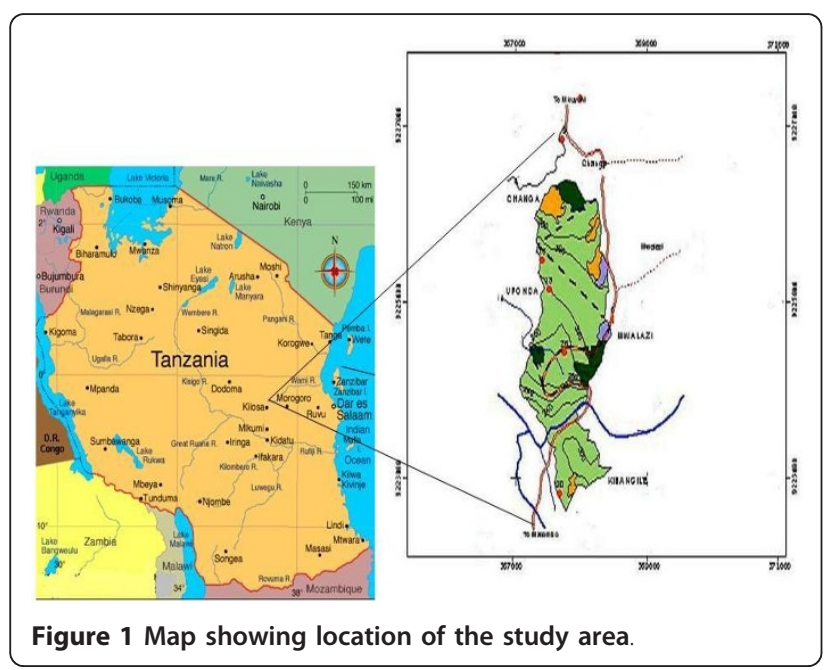

assistance of key respondents. Information regarding the local names of the plant species, medicinal uses, parts used, methods of preparation, and administration route were documented. The methods used in harvesting the plant materials from the wild were also recorded. Plant specimens were prepared, identified and voucher specimens deposited in the University of Dar es Salaam herbarium for future reference. Descriptive statistics were used to analyze the ethnomedicinal data collected.

\section{Results and discussion}

A total of 22 traditional medical practitioners were interviewed, with their ages ranging between 28 to 70 years, and $60 \%$ of them being older than 50 years. Out of the 22 informants interviewed, 18 of them were men and only four were women. A total of 82 medicinal plant species in 29 families were recorded as being used to treat 41 different types of diseases as shown in Table 1. The family Fabaceae had the highest proportion of medicinal plants used (29\%), followed by Euphorbiaceae (20\%), Asteraceae and Moraceae (17\% each), and Rubiaceae $(15 \%)$ in that order (Figure 2). Each of all other families had less than six plant species associated with the treatment of the diseases documented in Table 1.

At least $65.9 \%$ of all medicinal plants recorded were collected from the wild while only $26.7 \%$ were collected from cultivated land. The most frequently utilized medicinal plant parts were leaves $(41.3 \%)$, followed by roots (29.0\%), bark $(21.7 \%)$, seeds $(5.3 \%)$, and fruits $(2.6 \%)$ as shown in Figure 3. Roots were mostly used in the treatment of stomachache, diarrhea and inflammatory diseases, while leaves were mostly used in the treatment of malaria, skin diseases and children's diseases.

Most of the plant species were used to treat one disease, while some were used to treat two or more diseases. The plant species used to treat the highest percentage of diseases were Azadirachta indica A. Juss. and Bridelia micrantha (Hochst) Baill. each reported to treat $14.6 \%$ of the diseases. Ficus exasperate Valh., Mangifera indica L. and Senna hirsuta (L.) Irwin \& Barneby. were each reported to treat $12.2 \%$ of the diseases. The third category of highly used plant species were Ocotea usambarensis Engl. and Vernonia hymenolopis A. Rich. each reported to treat $9.8 \%$ of the diseases (Figure 4 ). In terms of frequency of medicinal plant uses, the highest percentage of plant species (15\%) was used to treat stomachache, followed by diarrhea (13\%) and hernia (12\%), fever and wound (11\% each) and cough (10\%). Other diseases were treated with less than $10 \%$ of the medicinal plants recorded (Figure 5).

Majority of the plant remedies were prepared by boiling $(44 \%)$, then by crushing or pounding (27\%), or soaking in cold water (19\%) and concoction 10\% (Figure 6). Some specific herbal preparations were taken by mixing 
Table 1 Medicinal plants used for treating human diseases in villages around Kimboza forest reserve

\begin{tabular}{|c|c|c|c|c|c|c|c|}
\hline Family/Botanical name & Local name & $\begin{array}{l}\text { Habitat/ } \\
\text { Source }\end{array}$ & $\begin{array}{l}\text { Part } \\
\text { used }\end{array}$ & Health problems cured & $\begin{array}{l}\text { Methods of } \\
\text { preparation }\end{array}$ & $\begin{array}{l}\text { Administration } \\
\text { route }\end{array}$ & $\begin{array}{l}\text { Voucher } \\
\text { No. }\end{array}$ \\
\hline \multicolumn{8}{|l|}{ Acanthaceae } \\
\hline $\begin{array}{l}\text { Justicia heterocarpa } \\
\text { L. }\end{array}$ & Mwidu & $\begin{array}{l}\text { Wild or } \\
\text { Cultivated }\end{array}$ & $\begin{array}{l}\text { Roots, } \\
\text { Leaves }\end{array}$ & Snake bite & $\begin{array}{l}\text { Crushed or } \\
\text { pounded }\end{array}$ & Oral & EA031 \\
\hline \multicolumn{8}{|l|}{ Alliaceae } \\
\hline $\begin{array}{l}\text { Allium ascalonium } \\
\text { L. }\end{array}$ & Kitunguu saumu & Cultivated & $\begin{array}{l}\text { Roots, } \\
\text { Leaves }\end{array}$ & Antifungal, Child fever & Decoction & Oral & EA040 \\
\hline Allium sativum $\mathrm{L}$. & Kitunguu maji & Cultivated & Leaves & $\begin{array}{l}\text { Fever, Cough, Asthma, } \\
\text { Malaria, Hypertension }\end{array}$ & Concoction & Oral & EA017 \\
\hline \multicolumn{8}{|l|}{ Anacardiaceae } \\
\hline $\begin{array}{l}\text { Sorindeia } \\
\text { madagascariensis DC. }\end{array}$ & Mpilipili & Wild & Leaves & Wound & $\begin{array}{l}\text { Crushed or } \\
\text { pounded }\end{array}$ & Topical & EA021 \\
\hline Mangifera indica $\mathrm{L}$. & Mwembe & Cultivated & $\begin{array}{l}\text { Leaves, } \\
\text { Bark, } \\
\text { Roots }\end{array}$ & $\begin{array}{l}\text { Malaria,Diarrhea, Hypertension, } \\
\text { Asthma, Cough }\end{array}$ & Decoction & Oral & EA025 \\
\hline \multicolumn{8}{|l|}{ Annonaceae } \\
\hline $\begin{array}{l}\text { Annona senegalensis } \\
\text { Pers. }\end{array}$ & Mtopetope & Wild & $\begin{array}{l}\text { Roots } \\
\text { Leaves }\end{array}$ & Eye ache, Wound & $\begin{array}{l}\text { Crushed or } \\
\text { pounded }\end{array}$ & Topical & EA035 \\
\hline \multicolumn{8}{|l|}{ Apocynaceae } \\
\hline $\begin{array}{l}\text { Landolphia } \\
\text { buchananii P.Beauv. }\end{array}$ & Luziwana & Wild & Roots & Stomach ache, Diarrhea, Hernia & Decoction & Oral & EA022 \\
\hline \multicolumn{8}{|l|}{ Asteraceae } \\
\hline $\begin{array}{l}\text { Vernonia iodocalyx } \\
\text { O. Hoffm. }\end{array}$ & Kitugutu & Wild & $\begin{array}{l}\text { Bark } \\
\text { leaves }\end{array}$ & Stomach ache, Diarrhea, Head ache & $\begin{array}{l}\text { Crushed or } \\
\text { pounded }\end{array}$ & Oral & EA010 \\
\hline $\begin{array}{l}\text { Helichrysum } \\
\text { schimperi (Sch. Bip. } \\
\text { ex A. Rich.) Moeser }\end{array}$ & Lweza & Wild & Roots & Stomach ache, Diarrhea, & Decoction & Oral & EA013 \\
\hline $\begin{array}{l}\text { Vernonia } \\
\text { hymenolepis A. Rich. }\end{array}$ & Msungu & Wild & $\begin{array}{l}\text { Roots, } \\
\text { Leaves }\end{array}$ & $\begin{array}{l}\text { Fever, Diarrhea, Hernia, Spleen } \\
\text { enlargement. }\end{array}$ & Decoction & Oral & EA006 \\
\hline $\begin{array}{l}\text { Crassocephalum } \\
\text { Montuosum (S. } \\
\text { Moore) Milne-Redh. }\end{array}$ & Nyaluganza & Wild & Bark & Ear ache, Head ache, burn & $\begin{array}{l}\text { Crushed or } \\
\text { pounded }\end{array}$ & Oral & EA004 \\
\hline Bidens pilosa $\mathrm{L}$. & Nyaweza & $\begin{array}{l}\text { Wild, } \\
\text { Cultivated }\end{array}$ & Bark & Wound, Spleen enlargement & Decoction & Topical & EA003 \\
\hline $\begin{array}{l}\text { Sonchus pinnatifidus } \\
\text { L. }\end{array}$ & Sungasunga & Wild & $\begin{array}{l}\text { Roots, } \\
\text { Leaves }\end{array}$ & Stomachache, Headache. & Decoction & Oral & EA032 \\
\hline Helianthus annus L. & Ufuta & Cultivated & Leaves & Chest pain, Asthma & Concoction & Oral & EA002 \\
\hline \multicolumn{8}{|l|}{ Asphodelaceae } \\
\hline Aloe vera (L.) Burm.f. & Mlovera & Cultivated & Leaves & Skin diseases, Wounds & $\begin{array}{l}\text { Crushed or } \\
\text { pounded }\end{array}$ & Topical & EA041 \\
\hline \multicolumn{8}{|l|}{ Bignoniaceae } \\
\hline $\begin{array}{l}\text { Kigelia africana (Lam.) } \\
\text { Benth. }\end{array}$ & Mwegea & Wild & $\begin{array}{l}\text { Bark, } \\
\text { Leaves, } \\
\text { Fruits }\end{array}$ & $\begin{array}{l}\text { Skin diseases, Venereal diseases, } \\
\text { Fever, }\end{array}$ & $\begin{array}{l}\text { Crushed or } \\
\text { pounded }\end{array}$ & Oral & EA026 \\
\hline \multicolumn{8}{|l|}{ Bombacaceae } \\
\hline $\begin{array}{l}\text { Adansonia digitata } \\
\mathrm{L} \text {. }\end{array}$ & Mbuyu & Wild & Roots & Hernia & Decoction & Oral & EA018 \\
\hline $\begin{array}{l}\text { Bombax } \\
\text { rhodognaphalon } \mathrm{L} \text {. }\end{array}$ & Msufipori & Wild & Leaves & Stomach ache & Decoction & Topical & EA024 \\
\hline \multicolumn{8}{|l|}{ Caricaceae } \\
\hline Carica papaya L. & Mpapai & Cultivated & $\begin{array}{l}\text { Roots, } \\
\text { Leaves }\end{array}$ & Tooth ache, Stomach-ache. & Decoction & Oral & EA015 \\
\hline \multicolumn{8}{|l|}{ Combretaceae } \\
\hline $\begin{array}{l}\text { Cobretum molle R.Br. ex } \\
\text { G.Don. }\end{array}$ & Mlamweusi & Wild & $\begin{array}{l}\text { Roots, } \\
\text { Leaves }\end{array}$ & Wounds, Cough, Malaria & Decoction & Oral & EA012 \\
\hline Terminalia sericea $\mathrm{L}$. & Mpululu & Wild & $\begin{array}{l}\text { Leaves, } \\
\text { Roots }\end{array}$ & Antifungal treatment & $\begin{array}{l}\text { Crushed } \\
\text { and } \\
\text { pounded }\end{array}$ & Topical & EA053 \\
\hline
\end{tabular}


Table 1 Medicinal plants used for treating human diseases in villages around Kimboza forest reserve (Continued)

\begin{tabular}{|c|c|c|c|c|c|c|c|}
\hline \multicolumn{8}{|l|}{ Cucurbitaceae } \\
\hline $\begin{array}{l}\text { Momordica foetida } \\
\text { L. }\end{array}$ & Huluhundu & Cultivated & Leaves & Malaria, Ear ache, Head ache, & Decoction & Oral & EA048 \\
\hline $\begin{array}{l}\text { Cucurbita moschata } \\
\text { Duchesne. }\end{array}$ & Maboga & Cultivated & Roots & Expulsion of placenta & Infusion & Oral & EA042 \\
\hline \multicolumn{8}{|l|}{ Euphorbiaceae } \\
\hline $\begin{array}{l}\text { Acalypha fruticosa } \\
\text { Forssk. }\end{array}$ & Kifulwe & Wild & Leaves & Diarrhea & Decoction & Oral & EA028 \\
\hline Jatropha curcas L. & Mbono & Wild & $\begin{array}{l}\text { Leaves } \\
\text { Seeds }\end{array}$ & Wound, Scabies & $\begin{array}{l}\text { Crushed } \\
\text { and } \\
\text { pounded }\end{array}$ & Topical & EA034 \\
\hline Fluggea virosa Willd. & Mkalananga & Wild & Leaves & $\begin{array}{l}\text { Stomach ache, Diarrhea, Hernia, } \\
\text { Spleen enlargement }\end{array}$ & Infusion & Oral & EA029 \\
\hline $\begin{array}{l}\text { Manihot esculenta } \\
\text { Crantz. }\end{array}$ & Mhogo & Cultivated & Leaves & Stomach ache & Infusion & Oral & EA043 \\
\hline $\begin{array}{l}\text { Suregada } \\
\text { zanzibariensis Roxb. } \\
\text { ex Rottler. }\end{array}$ & Mndimu pori & Wild & Roots & Malaria, Fever & Decoction & Oral & EA050 \\
\hline Ricinus communis L. & Mnyonyo & Wild & Leaves & Rheumatism, Wound & $\begin{array}{l}\text { Crushed } \\
\text { and } \\
\text { pounded }\end{array}$ & Topical & EA055 \\
\hline $\begin{array}{l}\text { Bridelia micrantha } \\
\text { (Hochst.) Baill. }\end{array}$ & Msumba & Wild & $\begin{array}{l}\text { Bark } \\
\text { Leaves }\end{array}$ & $\begin{array}{l}\text { Rheumatism, Hernia, Stomach ache, } \\
\text { Spleen enlargement, Skin eruption, } \\
\text { Insecticide }\end{array}$ & Decoction & Oral & EA036 \\
\hline $\begin{array}{l}\text { Euphorbia nyikae Pax ex } \\
\text { Engl. }\end{array}$ & Mngwede & Wild & Leaves & Wound & $\begin{array}{l}\text { Crushed } \\
\text { and } \\
\text { pounded }\end{array}$ & Topical & EA044 \\
\hline \multicolumn{8}{|l|}{ Fabaceae } \\
\hline $\begin{array}{l}\text { Cassia mimosoides } \\
\text { L. }\end{array}$ & Lusangalala & Wild & $\begin{array}{l}\text { Roots } \\
\text { Bark }\end{array}$ & $\begin{array}{l}\text { Mental illness, Asthma, Severe } \\
\text { cough }\end{array}$ & Decoction & Oral & EA056 \\
\hline $\begin{array}{l}\text { Senna petersiana } \\
\text { (Bolle) Lock. }\end{array}$ & Mkunde & Wild & $\begin{array}{l}\text { Roots } \\
\text { Leaves }\end{array}$ & $\begin{array}{l}\text { Skin diseases, Inflammation } \\
\text { Backache, Stomach ache, Skin } \\
\text { eruption }\end{array}$ & Infusion & Oral & EA054 \\
\hline $\begin{array}{l}\text { Senna hirsuta (L.) } \\
\text { Irwin \& Barneby. }\end{array}$ & Mwambalasimba & Wild & Leaves & $\begin{array}{l}\text { Pneumonia, Hernia, Stomach ache, } \\
\text { Head ache }\end{array}$ & Decoction & Oral & EA052 \\
\hline $\begin{array}{l}\text { Brachystegia } \\
\text { spiciformis Benth. }\end{array}$ & Mzinghawa nyika & Wild & Roots & Ear ache, Child fever. & Infusion & Oral & EA061 \\
\hline $\begin{array}{l}\text { Albizia versicola } \\
\text { Welw. ex Oliv. }\end{array}$ & Mkenge maji & Wild & $\begin{array}{l}\text { Roots } \\
\text { Bark }\end{array}$ & Skin diseases, Boils & $\begin{array}{l}\text { Crushed or } \\
\text { pounded }\end{array}$ & Topical & EA057 \\
\hline $\begin{array}{l}\text { Albizia petersiana } \\
\text { Oliv. }\end{array}$ & Mkenge poli & Wild & $\begin{array}{l}\text { Leaves } \\
\text { Bark }\end{array}$ & Skin diseases & $\begin{array}{l}\text { Crushed or } \\
\text { pounded }\end{array}$ & Topical & EA063 \\
\hline $\begin{array}{l}\text { Mucuna pruriens (L.) } \\
\text { DC }\end{array}$ & Bumu & Wild & Roots & Male fertility & Infusion & Oral & EA066 \\
\hline $\begin{array}{l}\text { Tephrosia vogelii } \\
\text { Hook.f. }\end{array}$ & Kitupa & Wild & Bark & Insecticide & $\begin{array}{l}\text { Crushed or } \\
\text { pounded }\end{array}$ & Topical & EA068 \\
\hline Abrus precatorius $\mathrm{L}$. & Lufambo & Wild & Roots & $\begin{array}{l}\text { Eye inflammation, Diarrhea, Women } \\
\text { fertility }\end{array}$ & Decoction & Oral & EA078 \\
\hline $\begin{array}{l}\text { Cajanus cajan (L.) } \\
\text { Millsp. }\end{array}$ & Mbaazi & Cultivated & Leaves & Diarrhea. & $\begin{array}{l}\text { Crushed or } \\
\text { pounded }\end{array}$ & Oral & EA071 \\
\hline $\begin{array}{l}\text { Vigna unguiculata } \\
\text { (L.) Walp. }\end{array}$ & Mkunde & Cultivated & $\begin{array}{l}\text { Roots } \\
\text { Leaves }\end{array}$ & Chest pain, Cough, Abscess, Hernia & Infusion & Oral & EA062 \\
\hline $\begin{array}{l}\text { Pterocarpus } \\
\text { angolensis DC. }\end{array}$ & Mninga & Wild & Bark & Hernia & Decoction & Oral & EA064 \\
\hline \multicolumn{8}{|l|}{ Lamiaceae } \\
\hline $\begin{array}{l}\text { Satureja biflora (Buch.- } \\
\text { Ham.ex D.Don) Briq. }\end{array}$ & $\begin{array}{l}\text { Luparalwa } \\
\text { mlungu }\end{array}$ & Wild & Leaves & Mental illness & Infusion & Oral & EA011 \\
\hline $\begin{array}{l}\text { Ocimum suave } \\
\text { Willd. }\end{array}$ & Mnung'ha & Wild & Bark & Malaria, Stomach ache, & Decoction & Oral & EA001 \\
\hline
\end{tabular}


Table 1 Medicinal plants used for treating human diseases in villages around Kimboza forest reserve (Continued)

\begin{tabular}{|c|c|c|c|c|c|c|c|}
\hline \multicolumn{8}{|l|}{ Lauraceae } \\
\hline $\begin{array}{l}\text { Ocotea } \\
\text { usambarensis Engl. }\end{array}$ & Mseli & Wild & $\begin{array}{l}\text { Roots } \\
\text { Bark }\end{array}$ & $\begin{array}{l}\text { Stomach ache, Fever, Malaria, } \\
\text { Hernia, Sprit }\end{array}$ & Infusion & Oral & EA009 \\
\hline \multicolumn{8}{|l|}{ Malvaceae } \\
\hline $\begin{array}{l}\text { Hibiscus surattensis } \\
\mathrm{L} \text {. }\end{array}$ & Lumotomoto & $\begin{array}{l}\text { Wild } \\
\text { Cultivated }\end{array}$ & Leaves & $\begin{array}{l}\text { Wound, } \\
\text { Abscess, Gonorrhea }\end{array}$ & $\begin{array}{l}\text { Crushed or } \\
\text { pounded }\end{array}$ & Topical & EA059 \\
\hline $\begin{array}{l}\text { Hibiscus fuscus } \\
\text { Garcke }\end{array}$ & Luswagamene & Wild & Roots & Rheumatism, Mental illness. & Concoction & Oral & EA043 \\
\hline \multicolumn{8}{|l|}{ Melastomataceae } \\
\hline $\begin{array}{l}\text { Dissotis rotundifolia } \\
\text { (Sm.) Triana. }\end{array}$ & Kinzasu & Wild & $\begin{array}{l}\text { Roots, } \\
\text { Leaves }\end{array}$ & $\begin{array}{l}\text { Wound, Asthma, Boil, Abscess } \\
\text { Diarrhea, Gonorrhea }\end{array}$ & $\begin{array}{l}\text { Crushed or } \\
\text { pounded; } \\
\text { Decoction }\end{array}$ & $\begin{array}{l}\text { Topical; } \\
\text { Oral }\end{array}$ & EA039 \\
\hline \multicolumn{8}{|l|}{ Meliaceae } \\
\hline $\begin{array}{l}\text { Khaya anthotheca } \\
\text { (Welw.) C. DC }\end{array}$ & Mkangazi & Wild & $\begin{array}{l}\text { Bark } \\
\text { Leaves }\end{array}$ & Malaria, Bilharzias, Gonorrhea & Concoction & Oral & EA067 \\
\hline $\begin{array}{l}\text { Azadirachta indica } \\
\text { A. Juss. }\end{array}$ & Mwarobaini & Cultivated & $\begin{array}{l}\text { Leaves, } \\
\text { Bark, } \\
\text { Seeds }\end{array}$ & $\begin{array}{l}\text { Head ache, Back ache, Malaria, } \\
\text { Fever, Stomach-ache, Insecticide }\end{array}$ & Decoction & Oral & EA080 \\
\hline Cedrella odorata $\mathrm{L}$. & Mwerezi & Wild & $\begin{array}{l}\text { Leaves } \\
\text { Bark }\end{array}$ & Menstrual cycle and Women fertility & Infusion & Oral & EA079 \\
\hline \multicolumn{8}{|l|}{ Moraceae } \\
\hline $\begin{array}{l}\text { Ficus altissima } \\
\text { Blume. }\end{array}$ & Mvira & Wild & $\begin{array}{l}\text { Bark } \\
\text { Leaves }\end{array}$ & Diarrhoea, Stomach-ache, & Concoction & Oral & EA077 \\
\hline $\begin{array}{l}\text { Ficus asperifolia Hook. ex } \\
\text { Steud. }\end{array}$ & Mkoya & Wild & $\begin{array}{l}\text { Roots } \\
\text { Leaves }\end{array}$ & Wounds & $\begin{array}{l}\text { Crushed or } \\
\text { pounded }\end{array}$ & Topical & EA075 \\
\hline Ficus exasperata Valh & Msasa & Wild & $\begin{array}{l}\text { Roots } \\
\text { Leaves } \\
\text { Bark }\end{array}$ & $\begin{array}{l}\text { Hypertension, Scabies, Stomach } \\
\text { disorders, Gonorrhoea, Diarrhea }\end{array}$ & Decoction & Oral & EA073 \\
\hline $\begin{array}{l}\text { Ficus stuhlmanii } \\
\text { Warb. }\end{array}$ & Foza/Mtamba & Wild & Bark & Stomach tumor & Infusion & Oral & EA069 \\
\hline Ficus sycomorus L. & Mkuyu & Wild & Bark & $\begin{array}{l}\text { Menstrual cycle, } \\
\text { Women fertility }\end{array}$ & Infusion & Oral & EA067 \\
\hline $\begin{array}{l}\text { Milicia excelsa } \\
\text { (Welw.) C.C Berg. }\end{array}$ & Mvule & Wild & Roots & Wound & $\begin{array}{l}\text { Crushed or } \\
\text { pounded }\end{array}$ & Topical & EA082 \\
\hline $\begin{array}{l}\text { Antiaris toxicaria } \\
\text { Lesch. }\end{array}$ & Mbila & Wild & $\begin{array}{l}\text { Leaves } \\
\text { Bark }\end{array}$ & Skin diseases, Insecticide. & $\begin{array}{l}\text { Crushed or } \\
\text { pounded }\end{array}$ & Topical & EA088 \\
\hline \multicolumn{8}{|l|}{ Moringaceae } \\
\hline $\begin{array}{l}\text { Moringa oleifera } \\
\text { Lam. }\end{array}$ & Mlonge & Cultivated & $\begin{array}{l}\text { Leaves } \\
\text { Bark } \\
\text { Seeds } \\
\text { Roots }\end{array}$ & $\begin{array}{l}\text { Skin diseases, headache, } \\
\text { [Detoxification, Rheumatism, } \\
\text { inflammation }\end{array}$ & $\begin{array}{l}\text { Decoction } \\
\text { or } \\
\text { Infusion }\end{array}$ & Oral & EA097 \\
\hline \multicolumn{8}{|l|}{ Myrtaceae } \\
\hline $\begin{array}{l}\text { Eucalyptus maidenii } \\
\text { Labill. }\end{array}$ & Mmaidini & $\begin{array}{l}\text { Wild } \\
\text { Cultivated }\end{array}$ & Bark & Chest pain, Cough & Decoction & Oral & EA087 \\
\hline Psidium guajava $\mathrm{L}$. & Mpera & Cultivated & Leaves & Diarrhea, Stomach ache & Infusion & Oral & EA085 \\
\hline \multicolumn{8}{|l|}{ Poaceae } \\
\hline Zea mays L. & Mahindi & Cultivated & Roots & Bedwetting & Decoction & Oral & EA091 \\
\hline $\begin{array}{l}\text { Saccharum } \\
\text { officinarum L. }\end{array}$ & Muwa & Cultivated & Roots & Bilharzias, Detoxifying kidneys & Decoction & Oral & EA101 \\
\hline \multicolumn{8}{|l|}{ Podocarpaceae } \\
\hline $\begin{array}{l}\text { Podocarpus latifolius } \\
\text { (Thunb.) R.Br. ex } \\
\text { Mirb. }\end{array}$ & Mnyanziri & Wild & Roots & Hernia & Decoction & Oral & EA99 \\
\hline \multicolumn{8}{|l|}{ Rosaceae } \\
\hline $\begin{array}{l}\text { Rubus pinnatus } \\
\text { Willd }\end{array}$ & Lufifi & Wild & Leaves & Menstrual cycle, & Infusion & Oral & EA093 \\
\hline
\end{tabular}


Table 1 Medicinal plants used for treating human diseases in villages around Kimboza forest reserve (Continued)

\begin{tabular}{|c|c|c|c|c|c|c|c|}
\hline $\begin{array}{l}\text { Prunus americana } \\
\text { Marshall. }\end{array}$ & Mpisi & Wild & Bark & Stomach ache & $\begin{array}{l}\text { Crushing } \\
\text { and water }\end{array}$ & Oral & EA090 \\
\hline \multicolumn{8}{|l|}{ Rubiaceae } \\
\hline $\begin{array}{l}\text { Multidentia fanshwei } \\
\text { (Tennant) Bridson. }\end{array}$ & Degedege & Wild & Roots & Child fever & Decoction & Oral & EA095 \\
\hline $\begin{array}{l}\text { Rytigynia } \\
\text { lichenixenos (K. } \\
\text { Schum.) Robyns. }\end{array}$ & Mhambalamaziwa & Wild & $\begin{array}{l}\text { Seeds } \\
\text { Roots }\end{array}$ & Hernia & Decoction & Oral & EA092 \\
\hline $\begin{array}{l}\text { Vangueria infausta } \\
\text { Burch. }\end{array}$ & Mviru & Wild & Seeds & Pneumonia, Menstrual cycle, & Infusion & Oral & EA084 \\
\hline $\begin{array}{l}\text { Rytigynia uhligii (K. } \\
\text { Schum. \& K.Krause) } \\
\text { Verdc. }\end{array}$ & Msanangare & Wild & Seeds & Hernia & Decoction & Oral & EA099 \\
\hline $\begin{array}{l}\text { Chassalia parvifolia } \\
\text { K. Schum. }\end{array}$ & Mguhu & Wild & Bark & Hernia, Chest pain, Malaria, & Concoction & Oral & EA102 \\
\hline $\begin{array}{l}\text { Catunaregum } \\
\text { spinosa (Thunb.) }\end{array}$ & Mtutuma & Wild & Leaves & Skin diseases & $\begin{array}{l}\text { Crushed or } \\
\text { pounded }\end{array}$ & Topic & EA104 \\
\hline \multicolumn{8}{|l|}{ Rutaceae } \\
\hline $\begin{array}{l}\text { Citrus limon (L.) } \\
\text { Burm.f. }\end{array}$ & Mlimau & Cultivated & Roots & Stomach ache & Decoction & Oral & EA103 \\
\hline $\begin{array}{l}\text { Zanthoxylum } \\
\text { deremense } \\
\text { (Engl.) }\end{array}$ & Mlungulungu & Wild & Fruits & Stomach ache, Child fever & Decoction & Oral & EA100 \\
\hline $\begin{array}{l}\text { Citrus aurantifolia } \\
\text { (Christm.) Swingle. }\end{array}$ & Mndimu & Cultivated & Roots & Gonorrheal, Diarrhea, & Decoction & Oral & EA117 \\
\hline \multicolumn{8}{|l|}{ Solanaceae } \\
\hline $\begin{array}{l}\text { Lycopersicum } \\
\text { esculentum Mill. }\end{array}$ & Mnyanya & Cultivated & $\begin{array}{l}\text { Roots, } \\
\text { Leaves }\end{array}$ & Stomach ache & Concoction & Oral & EA105 \\
\hline $\begin{array}{l}\text { Solanum incanum } \\
\text { L. }\end{array}$ & Mtula & $\begin{array}{l}\text { Wild } \\
\text { Cultivated }\end{array}$ & Leaves & Cough, Vomit. & Concoction & Oral & EA107 \\
\hline $\begin{array}{l}\text { Capsicum frutescens } \\
\text { L. }\end{array}$ & Pilipililukwale & Cultivated & $\begin{array}{l}\text { Roots, } \\
\text { Bark }\end{array}$ & Wound & $\begin{array}{l}\text { Crushed or } \\
\text { pounded }\end{array}$ & Topical & EA110 \\
\hline $\begin{array}{l}\text { Nicotiana tabacum } \\
\text { L. }\end{array}$ & Tumbaku & Cultivated & Fruits & Hernia & Decoction & Oral & EA106 \\
\hline \multicolumn{8}{|l|}{ Zingiberaceae } \\
\hline $\begin{array}{l}\text { Zingiber officinale } \\
\text { Roscoe. }\end{array}$ & Tangawizi & Cultivated & Roots & Cough & Decoction & Oral & EA111 \\
\hline
\end{tabular}
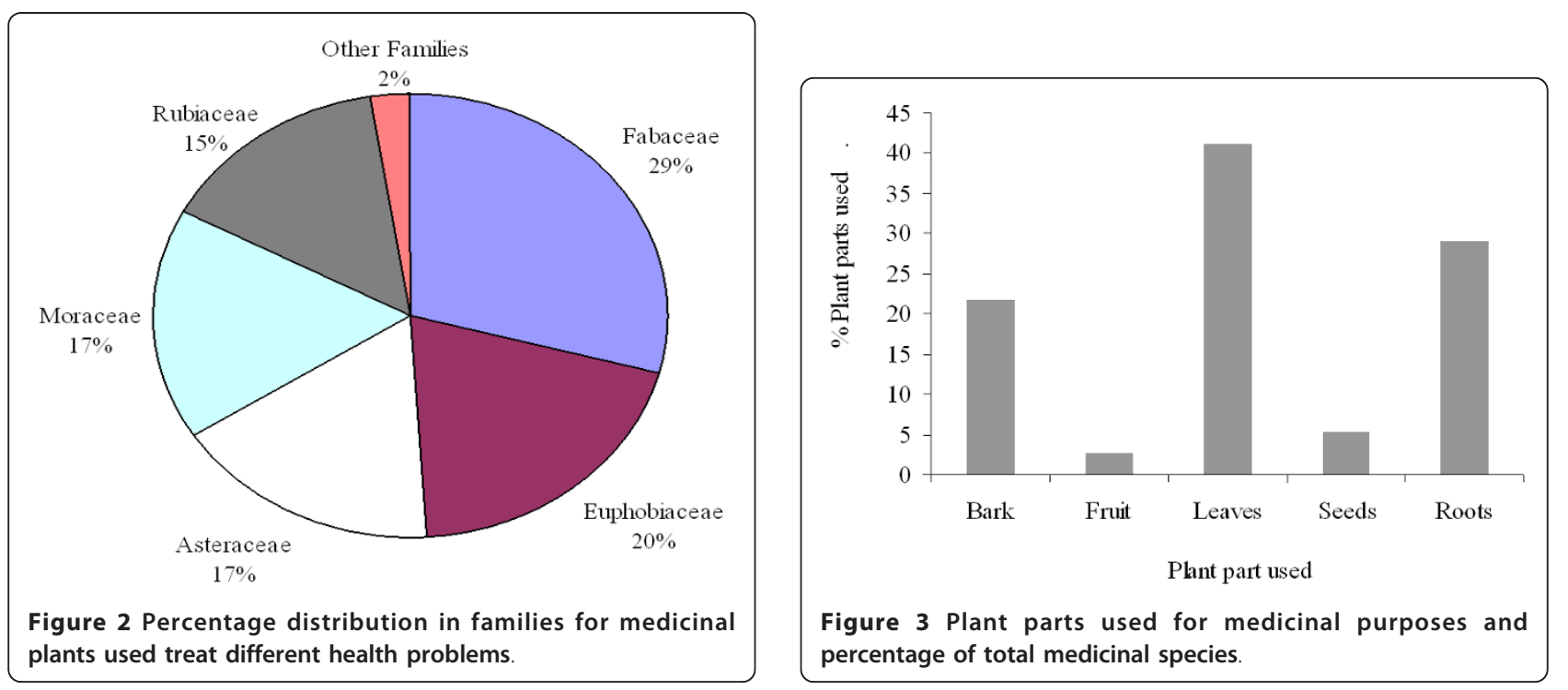


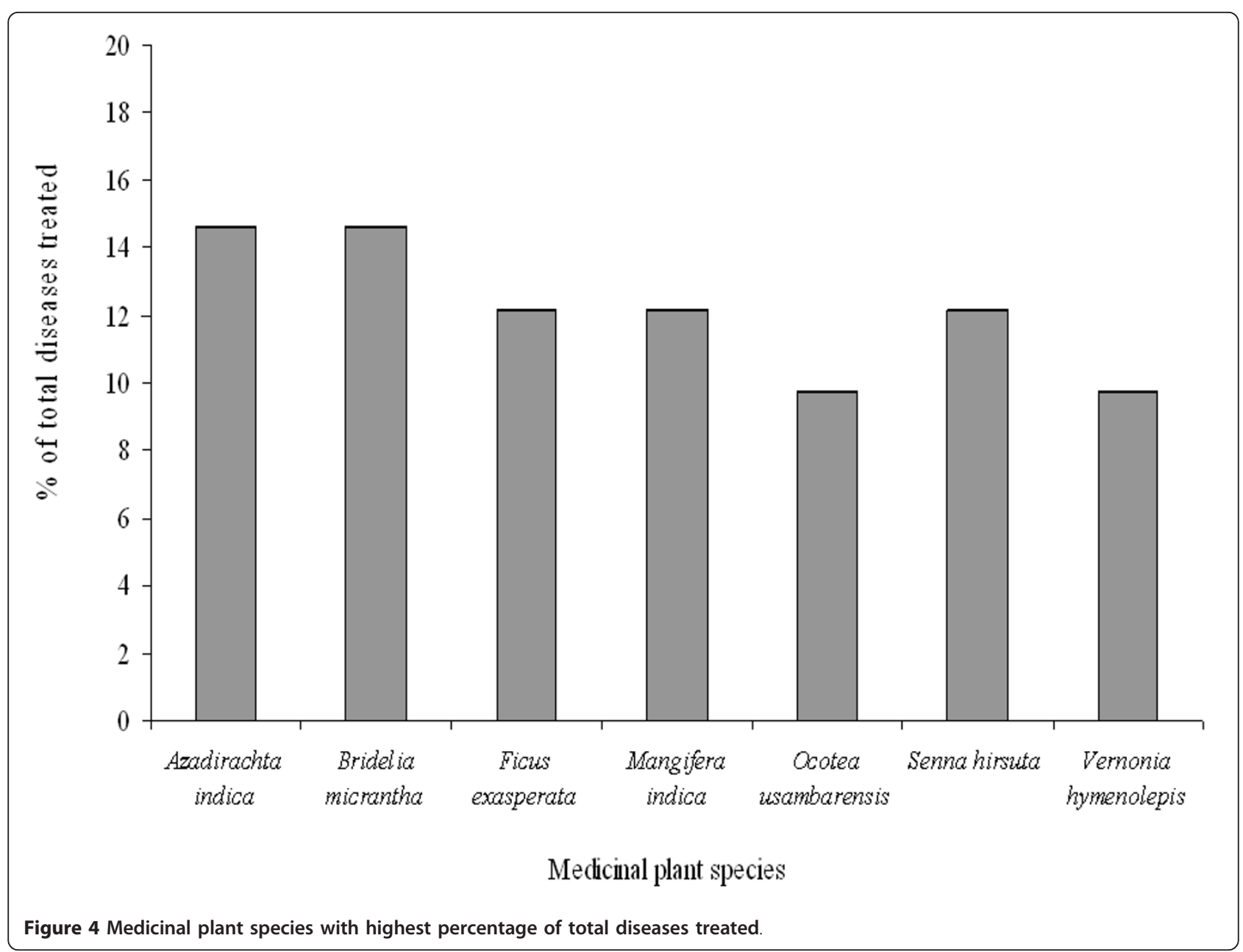

with food, honey or drunk together with coffee prepared from leaves of the coffee plant. Most medicinal plant preparations were taken orally $(75.6 \%)$, while $24.4 \%$ were administered topically for diseases such as skin infections and wounds. It was reported that different

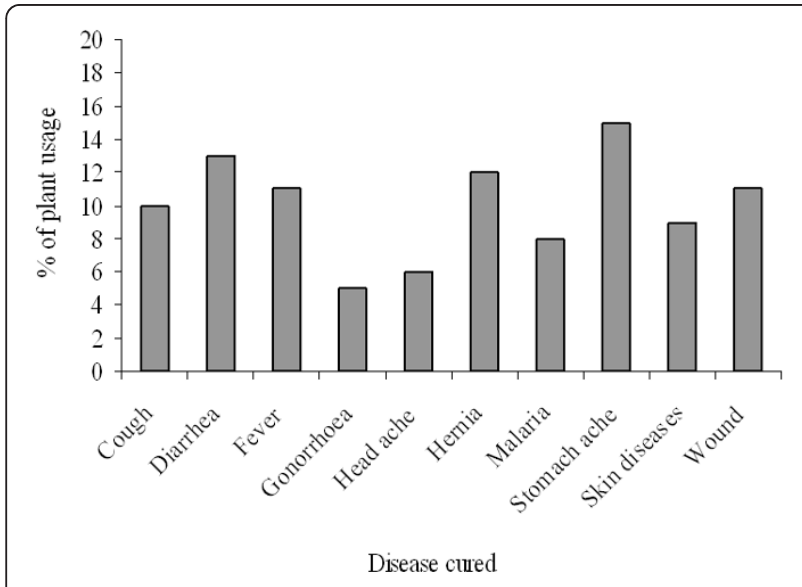

Figure 5 Frequency of medicinal plants uses to cure diseases. dosages were given to similar disease conditions. Administration dosage was by estimation and the amount of each dosage depended on the age of the patient and severity of the condition being treated.

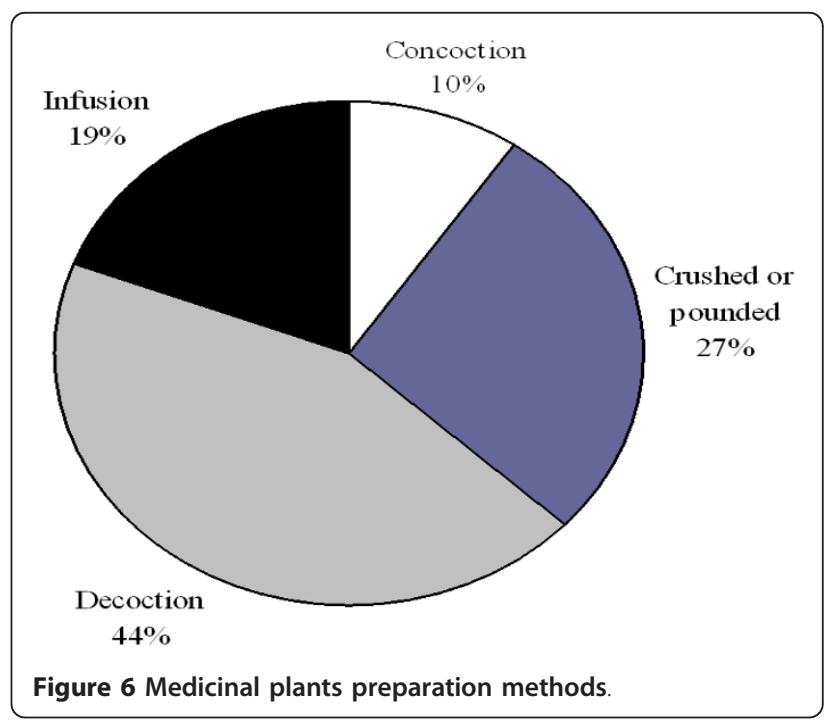


The study revealed a rich diversity of medicinal plants used to treat various disease conditions in the villages around Kimboza forest reserve. It further revealed a rich ethnobotanical knowledge amongst the residents of the neighbourhood of Kimboza Forest reserve. The families Fabaceae, Euphorbiaceae, Asteraceae, Moraceae and Rubiaceae which were the most dominant in this study are consistently recorded in other ethnomedicinal studies [9-12], and could be attributed to their wide range of bioactive ingredients $[13,14]$.

The fact that majority of the plant species recorded were sourced from the wild and only a few were cultivated may imply that many indigenous plant species may be difficult to propagate. This therefore calls for the need to train the herbal practitioners on the appropriate propagation techniques of these plant species for sustainable utilization. According to Edwards [15], about two-thirds of 50, 000 medicinal plants in use worldwide are still harvested from the natural habitat and about one fifth of them are now endangered. The increased percentage of species obtained from the wild has a direct effect on the availability of these resources and is likely to contribute to their vulnerability to being overexploited.

The finding that majority of the informants interviewed were aged above 50 years augments Kisangau et al. [16]. This implies that the elderly people are the main custodians of traditional knowledge, and this poses a serious challenge of the knowledge gap between the elderly and the young generation if framework to ensure apprenticeship is not put in place.

Some herbal practitioners reported that there was a potential to domesticate medicinal plants as some of them were already being planted on farmlands. Kisangau et al. [17] support the observation that only a few herbal practitioners were involved in cultivation of medicinal plants and most of them were gathered from the wild. The unabated over collection of the medicinal plants from the wild is a major threat to their existence and raises serious concern for their conservation. In the present study, the few plant species that were found to be cultivated on farmlands included Senna petersiana (Bolle) Lock., Azadirachta indica A. Juss., Khaya anthotheca (Welw.) C. DC. and Moringa oleifera Lam. However, unavailability of planting material and lack of appropriate propagation techniques were noted to be the major constraints to exploiting the potential for medicinal plant domestication. On the other hand, species like Carica papaya L., Vernonia iodocalyx O. Hoffm., Helichrysum schimperi (Sch. Bip. ex A. Rich) Moeser and Citrus aurantifolia (Christm) Swingle. could easily be conserved by planting them in home gardens.

It was found that the most commonly harvested plant parts were leaves followed by roots. These results are contrary with the findings of Rukia [18] who reported that roots were the most commonly harvested plant parts followed by leaves in Udzungwa Mountains Forests in Tanzania.

Some methods of harvesting medicinal plants like root excavation and bark striping can be very devastating and a big threat to the plant survival. The high utilisation of roots has also been reported as putting many plant species at a risk of extinction because of the damages inflicted on them in the course of uprooting them $[19,20]$. Bark striping is also an equally harmful harvesting method as reported for Prunus africana and other medicinal plants in Cameroon [7]. In Namibia just like in other countries, harvesting of roots and barks was found to be common, destructive and unsustainable [21].

The fact that the most frequently utilised plant parts were leaves is a more sustainable practice as opposed to where roots and/or the bark are used. The prevalence in the use of leaves for preparation of traditional herbal remedies has been reported in other studies too [9,22-26]. This practice helps to reduce the rate of threat on plant species and enhances the sustainable management of plants, as long as only an appreciable amount of leaves is harvested [27]. Leaves of plants have been reported to accumulate inulins, tannins and other alkaloids [28], which may be responsible for their various medicinal properties, hence explaining their wide use.

\section{Conclusion}

The results of the study revealed that there is rich diversity of medicinal plants used to treat various ailments in the neighbourhood of Kimboza forest reserve. Herbal practitioners and the local community in the study area should be educated on sustainable methods of harvesting medicinal plants without compromising their availability for future use. It is also imperative to train the community on the proper propagation techniques in order to encourage the domestication of valuable and threatened medicinal plants. The domestication of medicinal plants will create new opportunities for the local people such as provision of an alternative income and could help reduce the pressure on the wild population. Successful conservation strategies should be developed and priority given to sustainable harvesting of the plants.

\section{Acknowledgements}

EA is grateful for support from Rufford Small Grants on nature conservation which formed the basis of this work. The authors acknowledge the kindness and cooperation of the informants and local administrators in the study area, and the support of the University of Dar es Salaam Herbarium staff. EA and DPK also acknowledge the valuable input of Dr. Wycliffe Wanzala of South Eastern University College, Kenya in revising the manuscript. 


\section{Author details}

'Department of Science and Laboratory Technology, Dar es Salaam Institute of Technology, P. O. Box 2958, Dar es Salaam, Tanzania. ${ }^{2}$ Department of Biological Sciences, South Eastern University College (A Constituent College of the University of Nairobi) P.O Box 170-90200, Kitui, Kenya.

\section{Authors' contributions}

EA identified the research area and title, involved in field data collection, carried out statistical analysis and drafted the manuscript. DPK participated in refining data analysis and drafting as well as enrichment of the manuscript. All authors read, revised and approved the final manuscript.

\section{Competing interests}

The authors declare that they have no competing interest, and share the aspirations of the local people in the villages around Kimboza forest reserve to conserve medicinal plants for future generations.

Received: 20 February 2011 Accepted: 6 January 2012 Published: 6 January 2012

\section{References}

1. Pócs T: Bioclimatic studies in the Uluguru Mountains (Tanzania, East Africa). Acta Bot Acad Sci Hung 1976, 22:163-183.

2. Rodgers WA, Hall JB, Mwasumbi LB, Griffiths CJ, Vollesen K: The conservation values and status of Kimboza Forest Reserve, Tanzania. University of Dar es Salaam, mimeograph 1983, 84.

3. Schlage C, Mabula C, Mahunnah RLA, Heinrich M: Medicinal plants of the Washambaa (Tanzania): documentation and ethnopharmacological evaluation. Plant Biology 2000, 2:83-92.

4. WHO: Traditional medicine. Fact sheet No 1342003.

5. Hamayun M, Khan MA, Begum S: Marketing of medicinal plants of UtrorGabral Valleys, Swat, Pakistan. J Ethnobot leaflets SIUC, USA; 2003.

6. Muthu C, Ayyanar M, Raja N, Ignacimuthu S: Medicinal plants used be traditional healers in Kancheepuram District of Tamil Nadu, India. J Ethnobio Ethnomed 2006, 2:43.

7. Cunningham AB, Ayuk E, Franzel S, Duguma B, Asanga C: An economic evaluation of medicinal tree cultivation: Prunus africana in Cameroon. Peoples and Plants Working Paper 10 UNESCO, Paris; 2002.

8. Martin GJ: Ethnobotany: A people and plants conservation manual. London, UK: Chapman \& Hall; 1995.

9. Yineger $\mathrm{H}$, Yewhalaw D: Traditional Medicinal Plant Knowledge and Use by Local Healers in Sekoru District, Jimma Zone, Southwestern Ethiopia. J Ethnobiol Ethnomed 2007, 3:24.

10. Mesfin F, Demissew S, Teklehaymanot T: An ethnobotanical study of medicinal plants in Wonago Woreda, SNNPR, Ethiopia. J Ethnobiol Ethnomed 2009, 5:28

11. Moshi MJ, Otieno DF, Mbabazi PK, Weisheit A: Ethnomedicine of the Kagera Region, north western Tanzania. Part 2: The medicinal plants used in Katoro Ward, Bukoba District. J Ethnobiol Ethnomed 2009, 5:24

12. Ribeiro A, Maria M, Romeiras MM, Tavares J, Faria MT: Ethnobotanical survey in Canhane village, district of Massingir, Mozambique: medicinal plants and traditional knowledge. J Ethnobiol Ethnomed 2010, 6:33.

13. Gazzaneo LRS, Lucena RFP, Albuquerque UP: Knowledge and use of medicinal plants by local specialists in a region of Atlantic Forest in the state of Pernambuco (Northeastern Brazil). J Ethnobiol Ethnomed 2005, 1-9.

14. Simbo DJ: An ethnobotanical survey of medicinal plants in Babungo, Northwest Region, Cameroon. J Ethnobiol Ethnomed 2010, 6:8.

15. Edwards R: No remedy in sight for herbal ransack. New Science 2004, 181:10-11.

16. Kisangau DP, Lyaruu HVM, Hosea KM, Joseph CC: Use of traditional medicines in the management of HIV/AIDS opportunistic infections in Tanzania: a case in the Bukoba rural district. J Ethnobiol Ethnomed 2007, 3:29.

17. Kisangau DP, Herrmann TM, Lyaruu H, Hosea KM, Joseph CC, Mbwambo Z, Masimba P: Traditional Knowledge, Use Practices and Conservation of Medicinal Plants for HIV/AIDS Care in Rural Tanzania. J Ethnobot Res Appl 2011, 9:43-58.

18. Rukia AK: Use of medicinal plants for human health in Udzungwa Mountains Forests: a case study of New Dabaga Ulongambi Forest Reserve, Tanzania. J Ethnobiol Ethnomed 2007, 3:7.
19. Cunningham AB: Applied Ethnobotany; People, Wild Plant Use and Conservation. Earthscan Publishers Limited, London; 2001, 300.

20. Kamatenesi MM, Acipa A, Oryem-Origa H: Medicinal plants of Otwal and Ngai Sub Counties in Oyam District, Northern Uganda. J Ethnobiol Ethnomed 2011, 7:7.

21. Chinsembu KC, Hedimbi M: An ethnobotanical survey of plants used to manage HIV/AIDS opportunistic infections in Katima Mulilo, Caprivi region, Namibia. J Ethnobiol Ethnomed 2010, 6:25.

22. Asase A, Oteng-Yeboah AA, Odamtten GT, Simmonds MS: Ethnobotanical Study of Some Ghanaian Anti-Malarial Plants. J Ethnopharmacol 2005, 99(2):273-279.

23. Giday M, Teklehaymanot T, Animut A, Mekonnen Y: Medicinal plants of the Shinasha, Agew-awi and Amhara peoples inNorthwest Ethiopia. $J$ Ethnopharmacol 2007, 110:516-525.

24. Pradhan BK, Badola HK: Ethnomedicinal plant use by Lepcha tribe of Dzongu valley, bordering Khangchendzonga Biosphere Reserve, in North Sikkim, India. J Ethnobiol Ethnomed 2008, 4:22.

25. Focho DA, Newuh MC, Anjah MG, Nwana FA, Ambo FB: Ethnobotanical survey of trees in Fundong, Northwest Region, Cameroon. J Ethnobiol Ethnomed 2009, 5:17.

26. Signorini MA, Piredda M, Bruschi P: Plants and traditional knowledge: An ethnobotanical investigation on Monte Ortobene(Nuoro, Sardinia). J Ethnobiol Ethnomed 2009, 5:6.

27. Ayyanar M, Ignacimuthu S: Traditional knowledge of Kani tribals in Kouthalai of Tirunelveli hills, Tamil Nadu, India. J Ethnopharmacol 2005, 102(2):246-255.

28. Okoegwale EE, Omefezi JU: Some herbal preparations among the people of Isoko Clan of Delta State, Nigeria. J Appl Sci 2001, 4:2350-2371.

doi:10.1186/1746-4269-8-1

Cite this article as: Amri and Kisangau: Ethnomedicinal study of plants used in villages around Kimboza forest reserve in Morogoro, Tanzania. Journal of Ethnobiology and Ethnomedicine 2012 8:1.

\section{Submit your next manuscript to BioMed Central and take full advantage of:}

- Convenient online submission

- Thorough peer review

- No space constraints or color figure charges

- Immediate publication on acceptance

- Inclusion in PubMed, CAS, Scopus and Google Scholar

- Research which is freely available for redistribution

Submit your manuscript at www.biomedcentral.com/submit
Ciomed Central 